Data Descriptor

\title{
Short Baseline Observations at Geodetic Observatory Wettzell
}

\author{
Apurva Phogat *(1), Gerhard Kronschnabl, Christian Plötz, Walter Schwarz and Torben Schüler \\ Geodetic Observatory Wettzell, Federal Agency for Cartography and Geodesy, Sackenrieder Str. 25, \\ 93444 Bad Koetzting, Germany; gerhard.kronschnabl@bkg.bund.de (G.K.); christian.ploetz@bkg.bund.de (C.P.); \\ walter.schwarz@bkg.bund.de (W.S.); torben.schueler@bkg.bund.de (T.S.) \\ * Correspondence: apurva.phogat@bkg.bund.de; Tel.: +49-9941-603-105
}

Received: 15 November 2018; Accepted: 25 November 2018; Published: 10 December 2018

\begin{abstract}
The Geodetic Observatory Wettzell (GOW), jointly operated by the Federal Agency for Cartography and Geodesy (BKG), Germany and the Technical University of Munich, Germany is equipped with three radio telescopes for Very Long Baseline Interferometry (VLBI). Correlation capability is primarily designed for relative positioning of the three Wettzell radio telescopes i.e., to derive the local ties between the three telescopes from VLBI raw data in addition to the conventional terrestrial surveys. A computing cluster forming the GO Wettzell Local Correlator (GOWL) was installed in 2017 as well as the Distributed FX (DiFX) software correlation package and the Haystack Observatory Postprocessing System (HOPS) for fringe fitting and postprocessing of the output. Data pre-processing includes ambiguity resolution (if necessary) as well as the generation of the geodetic database and NGS card files with $v$ Solve. The final analysis is either carried out with local processing software (LEVIKA short baseline analysis) or with the Vienna VLBI and Satellite (VieVS) software. We will present an overview of the scheduling, correlation and analysis capabilities at GOW and results obtained so. The dataset includes auxiliary files (schedule and log files) which contain information about the participating antenna, observed sources, clock offset between formatter and GPS time, cable delay, meteorological parameters (temperature, barometric pressure, and relative humidity) and ASCII files created after fringe fitting and final analysis. The published dataset can be used by the researchers and scientists to further explore short baseline interferometry.
\end{abstract}

Dataset: http:/ /dx.doi.org/10.17632/t8wvwzmk59.1

Dataset License: CC BY 4.0

Keywords: VLBI; geodesy; FX correlator; radio telescope; local ties; short baseline interferometry; scheduling

\section{Summary}

This paper is intended to give the readers an insight into all the stages of data processing before and after a VLBI experiment. VLBI is a technique which is based on the computation of the time delay in the arrival of a radio frequency signal transmitted by a remote astronomical source (generally a quasar) between radio telescopes. At GOW, since 2017 two sibling VLBI telescopes (shown in Figure 1) which are 123 metre apart are used to make short baseline observations. The main idea of making such observations is to understand the difference between long and short baseline observations in terms of estimated parameters due to common troposphere and ionosphere and compare it with the results of local survey measurements [1]. Local sessions with short duration are also scheduled predominantly for research and development work conducted on the regular basis. These sessions are helpful in 
terms of quality affirmation at the VLBI sites and can provide quick feedback about the health of the system. To start planning any kind of local session whether experimental or geodetic it is very important to find common timeslots where both the telescopes are not participating in any of the long baseline observations. The VLBI telescopes at Wettzell [2] are currently participating in different types of sessions which are proposed by IVS [3] and to make this job easy a coordination tool [4] is developed. The coordination tool is useful for planning, coordinating, keeping track of the status of the session and statistical analysis. The principal investigator (P.I.) has to submit a request for the local session based on the available time interval for the baselines of interest using the tool.
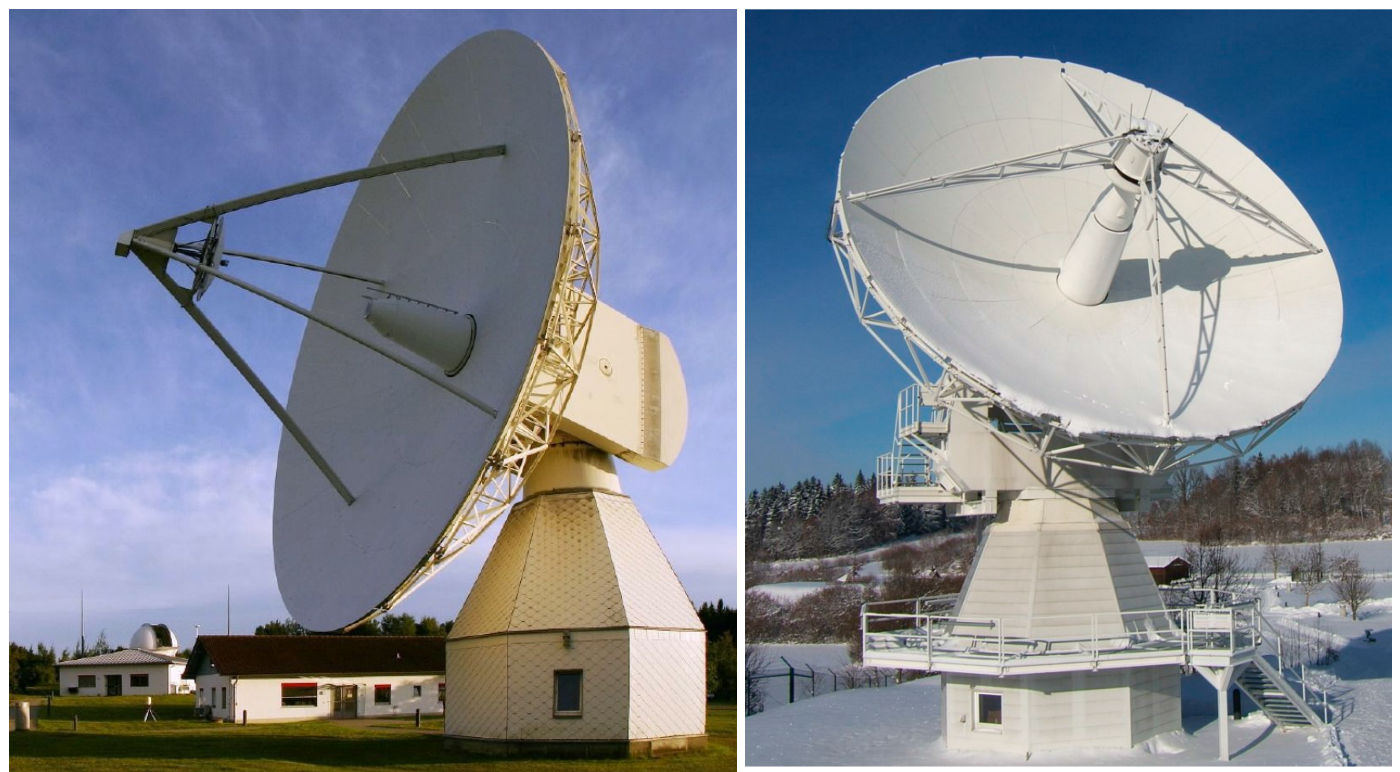

Figure 1. Image of Radio Telescope Wettzell (RTW) on the left and one of the twin telescopes in Wettzell (TTW1) on the right.

Table 1 provides statistics about all the different types of sessions observed in the year of 2017 by the two VLBI (legacy mode) telescopes from the GOW. Table 2 gives information about the technical specifications in terms of hardware installed at the telescopes [5,6]. Raw data from all the participating telescopes are sent to the correlation center either by an internet link or by shipping the hard disks. The correlation center is responsible for correlating and fringe fitting the observed data. A software correlator called DiFX [7,8] is currently used by most of the correlation centres around the globe. The fringe fitting software accounts for insufficiencies in the correlator model and integrates the interferometer signal. The software package called HOPS [9] is used for fringe fitting the correlated data. Fringe plots are helpful in detecting problems at the station and checking the quality of the data. A fringe plot is a collection of multiple plots on a page along with written information about a single scan. A description of the fringe plot for the analyst is given in Section 3.4. The fringe fitted data are further analysed in the postprocessing software for ambiguity resolution and ionospheric correction. The results are written in an ASCII file containing group delay information corresponding to each scan which is read by the in-house analysis software for the estimation of geodetic parameters, e.g., baseline vector and clock drift. The published dataset contains sessions observed with telescopes connected to separate clocks. 
Table 1. VLBI Session Distribution for 2017.

\begin{tabular}{cccc}
\hline Session Name & Wz & Wn & Total \\
\hline IVS-R1 & 47 & 26 & 73 \\
IVS-R4 & 49 & 27 & 76 \\
IVS-T2 & 7 & - & 7 \\
EUROPE & 2 & 2 & 4 \\
VLBA & 5 & 4 & 9 \\
R \& D & 14 & 4 & 18 \\
CONT & 15 & 15 & 30 \\
INT1 & 228 & 4 & 232 \\
INT2 & 101 & 2 & 103 \\
INT3 & 40 & 44 & 84 \\
GOW & 30 & 35 & 65 \\
Total & 538 & 163 & 701 \\
\hline
\end{tabular}

Table 2. Technical specifications of the participating telescopes.

\begin{tabular}{cccc}
\hline Antenna & Diameter $(\mathbf{m})$ & Front-End & Back-End \\
\hline $\mathrm{Wz}$ & 20 & Dual-band $(\mathrm{S} / \mathrm{X})$ & $\mathrm{DBBC}$ \\
$\mathrm{Wn}$ & 13.2 & Tri-band $(\mathrm{S} / \mathrm{X} / \mathrm{Ka})$ & $\mathrm{DBBC}$ \\
\hline
\end{tabular}

\section{Data Description}

The published dataset includes sessions observed in the year 2017 and 2018. From scheduling to analysis [10] all the different data types and file formats used in the VLBI processing will be described in this section. Scheduling is defined as the observation plan for the participating telescopes i.e., list of all the observed sources, source and station positions, observation epoch, duration of individual scans and additional information related to hardware. Two commonly used software (sked [11,12] and VieVS [13]) are installed for scheduling local (both long and short baseline) sessions. All the details about the file types are given in Section 2.1. VLBI measurement [14] takes place after the schedule is provided to all the network stations. The operators at each site use field system [15] to communicate with the hardware (mainly data acquisition system). To keep track of observation, field system generates log files where system check commands together with the operator comments are stored. Section 2.2 is dedicated to providing a brief overview of the useful information written in the log files during the measurement. The recorded raw data and the log files are transferred to the correlator facility. Further information related to the file formats generated at this step of data processing can be found in Section 2.3. The fringe fitted data are the input to the VLBI analysis software [16] developed for geodetic parameter estimation (EOP [17] and station coordinates [18] in particular). In Sections 2.4 and 2.5 descriptions of the NetCDF and ASCII files generated during the analysis process is given respectively.

\subsection{Schedule Files}

The Vienna VLBI and Satellite Software (VieVS) [19] is used for scheduling local sessions. For generating a schedule file, start time and duration of the requested session has to be inserted in addition to the list of participating stations. Two files are created as an output after providing all the required input information to the scheduling software i.e., sessionname.skd and sessionname.vex file.

\subsubsection{Skd File}

The schedule files with the extension .skd are mainly generated for the VLBI measurement purpose. These files are read by 'drudg' subroutine for producing snap and procedure files as an output for individual participating telescopes. A brief description of all the sections present in the skd file is given in Table 3. A detailed description of all the sections in a .skd file can be found in [20]. Procedure 
file contains information related to the hardware configuration, e.g., type of back-end, number of channels, bit-rate, local oscillator frequencies and other details, for instance, phase-Cal unit, baseband frequency with bandwidth and polarization. Snap file provides information about the position of the source at the time of observation for each network station allowing the antenna control unit to point in the right direction.

Table 3. Important sections with the description in the .skd file.

\begin{tabular}{cc}
\hline Section & Description \\
\hline \$EXPER & Experiment title \\
\$PARAM & Parameters used by sked and drudg \\
\$SOURCES & List of sources for this experiment \\
\$STATIONS & List of stations in this experiment \\
\$FLUX & Flux densities for each source \\
\$CODES & Frequency sequences and station LOs \\
\$HEAD & Tape recorder head position \\
\$PROC & Station procedures \\
\$OP & Automatic scheduling options \\
\$SKED & Scheduled observations \\
\hline
\end{tabular}

\subsubsection{VEX File}

The VEX stands for VLBI Experiment. The .vex file [21] is used for correlating the raw data after the session is observed. This file is either provided by the scheduler along with the .skd file to the correlation center or it is the responsibility of the correlation center to generate it. A vex file is a combination of snap and procedure file. Both source and hardware information essential for the correlation process is written in this file. It also contains frequency set-up and data recording rate set by the scheduler for a particular session. There are mainly three types of sections in a vex file. As shown in Figure 2, the blocks which are independently defined are in green. They are called primitive blocks. The high level blocks in pink are a combination of primitive-block keywords. The scheduling block in yellow contains a schedule for the measurement.

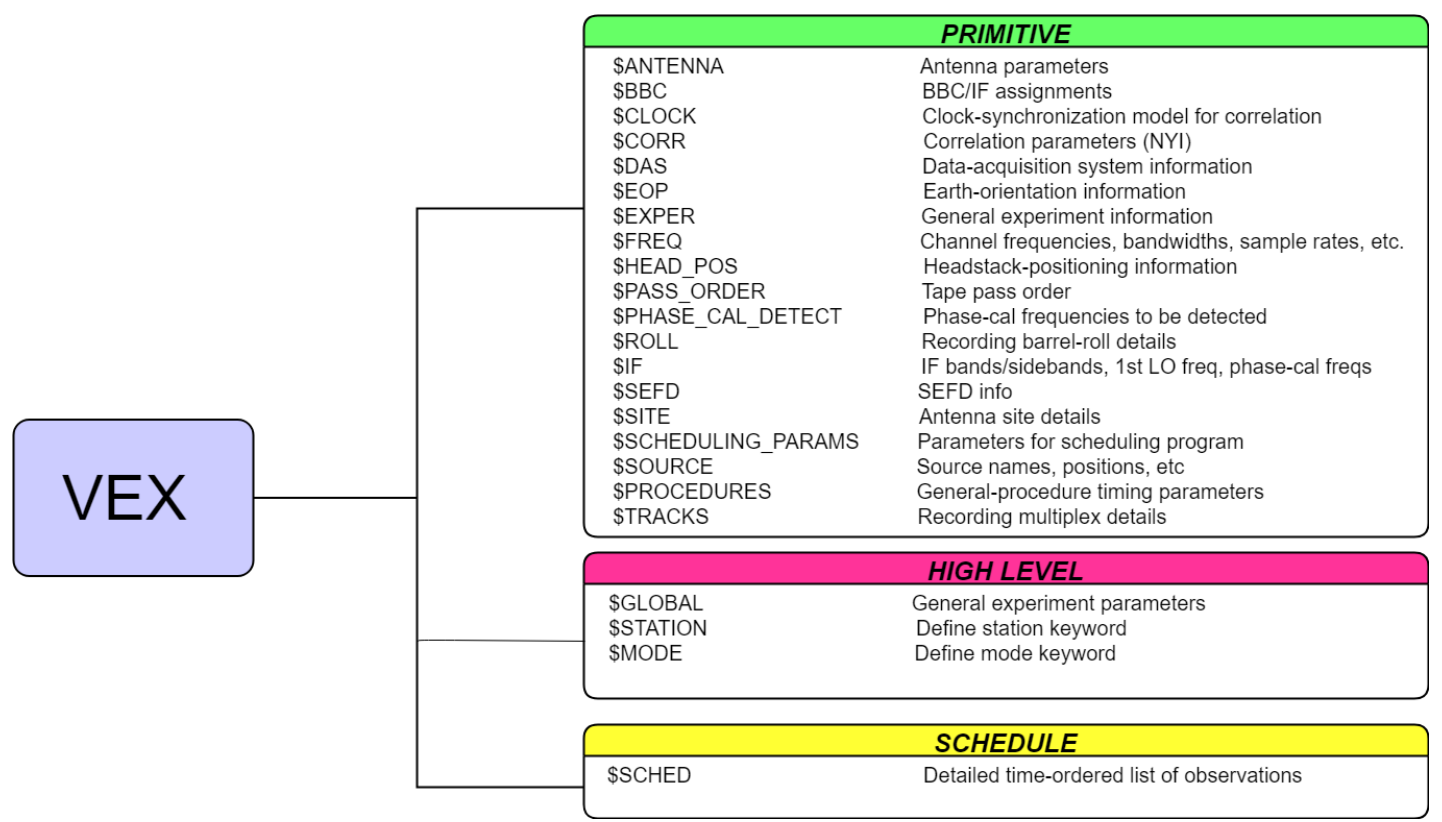

Figure 2. Important blocks with the description in a .vex file. 


\section{2. $\log$ File}

The scheduler has to send the schedule (.skd or .vex) file to all the participating stations where the operators create snap and procedure files in the Field System [22] (FS) using a program called 'drudg'. Every single snap instruction by the operator with the feedback is recorded in a log file. The log files provide auxiliary information about the session and are used in the correlation and analysis stage of data processing. Operator's comment section (Figure 3) also plays an important role in giving quick hint about the problems at the station to the data analyst.

$2018.136 .19: 15: 00.66: ! 2018.136 .19: 24: 58$

2018.136.19:15:02.95\#acusv\#Command Accepted from ACU

2018.136.19:15:03.35\#acusv\#Command Accepted from ACU

2018.136.19:15:08.17\#flagr\#flagr/antenna, acquired

2018.136.19:23:52.27;"Wettzell, we have a problem.

2018.136.19:24:26.06;"our ups batteries have overheating and we need to shut them down.

2018.136.19:24:46.48;"this means, we have to powerdown the whole VLBI system for some minutes. $2018.136 .19: 24: 58.00:$ preob

Figure 3. Screen shot from a log file where the operator is informing about the overheated UPS during the observation. These comments help correlation and analysis center in understanding the reasons for missing scans.

\subsubsection{Maser Offset}

For estimation of precise value and rate of change of UT1, it is important for the data analyst to know the offset between 1 pulse per second generated by the maser at the station and the GPS 1 pulse per second signal. In addition to UT1 determination, this value is also necessary at the correlation stage in adjusting the fringe search window. Each VLBI site has its own timing system and for the sake of uniformity, it is mandatory to record the offset between the standard/reference with the station clock. The offset counter is connected to the field system computer for registering the offset at least one time per scan [23]. The offset is stored in the log file during the session either after 'gps-fmout' or 'fmout-gps' command depending on the cable connection to the counter.

These values are also important in detecting clock jumps at the station. Figure $4 \mathrm{a}$ is an example without clock jump whereas Figure $4 \mathrm{~b}$ shows an example where WETTZELL telescope experienced clock jump during a 24-h VLBI session. All the scans in the red circle are observed after the clock jump and this has to be taken into consideration during the fringe search process.

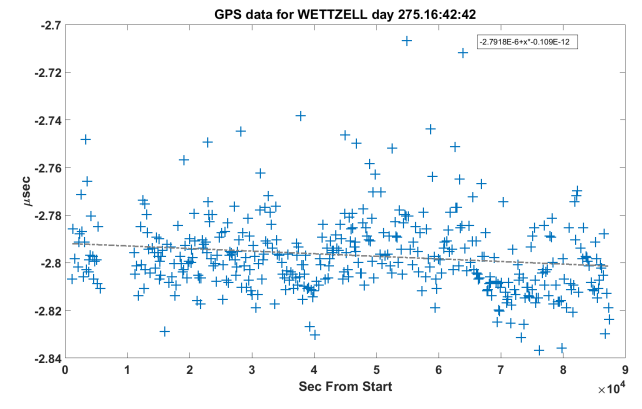

(a)

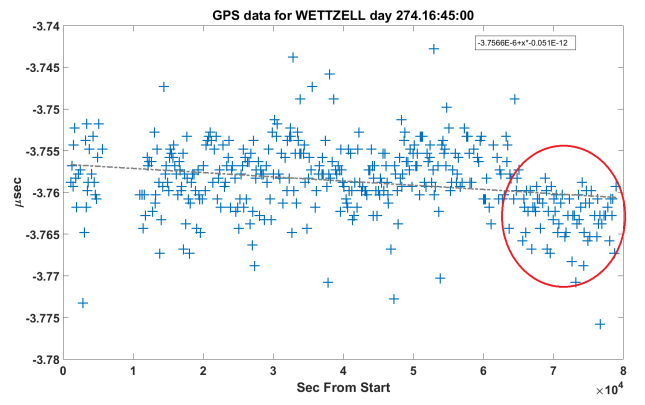

(b)

Figure 4. Clock-offset vs. observation time extracted from the log file for WETTZELL station. Offset values for each observed scan are represented in blue. The trend line is in grey. (a) Plot from 18OCT15XA; (b) Plot from 18OCT01XA.

\subsubsection{Cable Delay}

Cable delay system is installed for accounting the propagation delay caused by thermal or physical changes inside the VLBI system. Due to the presence of several electronic component inside the telescope, signal path monitoring plays an important role in estimating the instrumental delay [24]. 
Similar to clock offset, the calibration system is also connected to the field system computer and for each scan, a calibration signal is sent and received back. The values recorded by the cable delay system are written in the station log after 'cable' command. The cable delay values are used in the VLBI data analysis process. Cable delay values for WETTZELL station from the 18AUG02X9 session are shown in Figure 5.

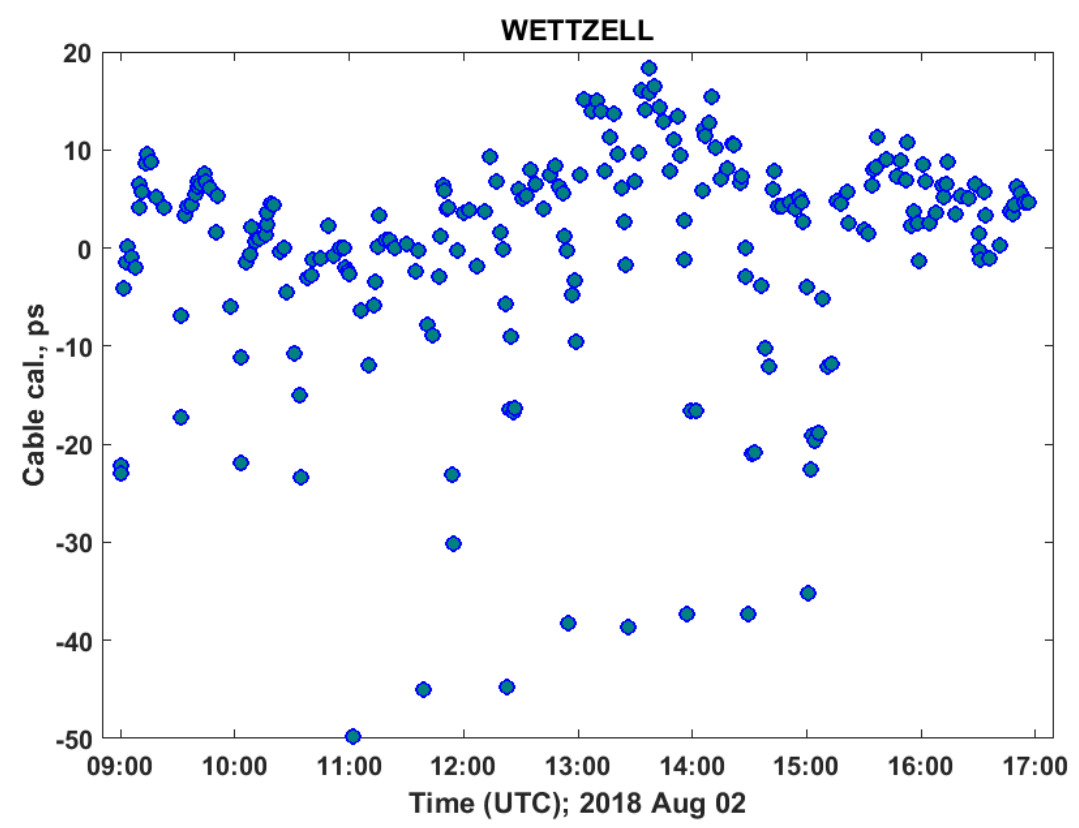

Figure 5. Cable delay versus time from a local session 18AUG02X9 for WETTZELL station.

\subsubsection{Meteorological Data}

For estimating additional delay caused by troposphere $[25,26]$ during the wave propagation in the VLBI processing chain, it is important to record temperature, pressure and relative humidity values for each station during the observation for each scan. These values are stored in the station log after ' $w x^{\prime}$ command and extracted by the analysis software for estimating tropospheric parameters (dry, wet delays and gradients). In the local sessions, the values for both the stations are recorded from the same meteorological sensor hence they are identical. In case of coordinate determination, short baseline observation shows more precise results as compared to long baseline observation. The main reason being, excess delay due to troposphere and ionosphere is close to negligible. Figure 6 shows recorded temperature, pressure and relative humidity values from session 18AUG02X9.

\section{3. alist.out}

Correlation and fringe fitting is performed at the correlation centre. The raw data are deleted after the analysis is finished to prevent disk space shortage. However, an ASCII file generated using HOPS [27] is stored for each correlated session. A file named alist.out contains scan-wise information about the session. This file is also used for creating a correlation report where session statistics is written with the correlation experts comment. List of important fields with the description in an alist.out is given in Table 4. 

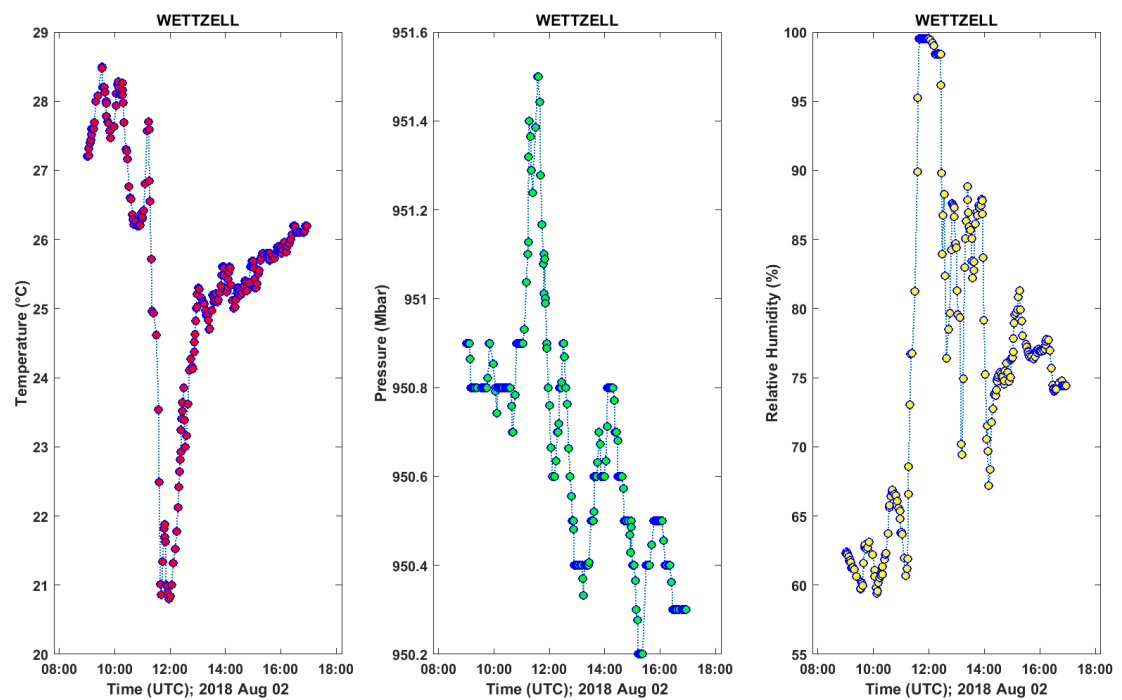

Figure 6. Meteorological dataset extracted from the NetCDF file. The dataset from both the telescopes are collected from the same sensor, hence the values are exactly the same for the other telescope as well.

Table 4. Important headers with the description in the alist.out file.

\begin{tabular}{cc}
\hline Header Name & Description \\
\hline ROOT & Root code \\
F\# & Frequency subgroup \\
DUR & Scan duration \\
EXP\# & Scan identifier \\
SCAN_ID & Year of observation \\
YEAR & Time in UTC \\
TIMETAGS & Source name \\
SOURCE & Baseline name given by 1 letter code \\
BS & Fringe quality code \\
Q & Frequency code with number of channels \\
FM\#C & Polarization \\
PL & Number of lags \\
LAGS & Signal to noise ratio \\
SNR & Single band delay \\
SBDLY & Multi-band delay \\
MBDLY & Delay rate \\
DRATE & Source elevation angle \\
ELEVATION & Source azimuth angle \\
AZIMUTH & Total phase \\
REF_FREQ & Reference frequency defined in the control file during fringe fitting \\
TPHASE & Total delay rate \\
TOTDRATE & Total multi-band delay \\
TOTMBDELAY & Total single band delay minus multi-band delay \\
TOTSBDMMBD & Coherence time \\
COHTIMES &
\end{tabular}

\subsection{NetCDF/ogos DB}

Database creation is the next step after the fringe fitting of the data. With the improvements in the technology and increasing demand, a new VLBI observation system called VGOS [28] is established. The main idea is to increase the observation type with broader bandwidth (2-14 GHz). So, to deal with data processing of VGOS style of observation [29], IVS working group 4 (WG4) [30] was established in 2007. Considering the fact that the amount of data will be high and it is important to have a file format which is easy to handle and know for the community, it was decided to use NetCDF as 
a standard file format. The fringe fitted output is input to the VLBI data analysis software [31] and the first database is generated which is NetCDF format. All the directories generated in the first database are listed with the description in Table 5. The data are split into pieces and stored in different folders to keep it flexible. A wrapper file is an ASCII file read by the software to locate specific data. They play a very important role in the whole data processing as they are never overwritten. It is very essential for testing different models and make comparisons. They also provide a lot of information and are stored with .wrp extension, e.g., 17SEP29X9_V001_kall.wrp where 17SEP29 is the session name, X9 is the session code and V001 is an indicator of version number which means, in this case, it is version 1.

Table 5. List of files/folders in a VGOS database directory.

\begin{tabular}{cc}
\hline File / Folder Name & Description \\
\hline Head.nc & General information about the session \\
sessionname_V001_kall.wrp & Wrapper file contains organisational information about the dataset \\
WETTZELL and WETTZ13N & Station related information is stored in these folders \\
Solve & Integer and fractional part of the time tag of scan \\
Session & Information about fourfit output \\
Scan & Information about the observed scan and correlation root file \\
Observables & Information about observables of the session \\
ObsCalTheo & Information about theoretical observables \\
History & Data processing history \\
Cross referencing & Cross referencing information between the observation and scan \\
\hline
\end{tabular}

Every time a session is processed, the version number incrementation takes place and a new wrapper file is created. The Head.nc file provides general information about the session, e.g., station list, source list and time of observation. Station folder contains station related information. Only two folders are available in case of this dataset i.e., WETTZELL and WETTZ13N. The start time of each scan can be found in Solve folder. Fourfit related information, e.g., control file and command string for each band are available in the sessions folder. Scan folder holds mainly all the information about the observed scan, e.g., scan names, a pointer to the fourfit output and observation time in UTC. The VLBI observables for each band are stored in the observables folder. A complete list with the description is available in Table 6.

Table 6. List of VLBI observables.

\begin{tabular}{cc}
\hline Observable Name & Description \\
\hline AmbigSize & Ambiguity spacing size \\
Baseline & Site names \\
Channel info & Local oscillator and amplitude and phase information \\
Correlation & Correlation coefficient \\
Corrinfo-difx & Fringe/fourfit related information \\
DataFlag & Database flagging information \\
GroupDelay & Group delay \\
GroupRate & Delay rate \\
Phase & Total phase \\
PhaseCalInfo & Pringe quality \\
QualityCode & calibration system details \\
RefFreq & Fringe fingling \\
SBDelay & Single band delay \\
SNR & signal to noise ratio \\
SOURCE & List of sources \\
TimeUTC & Time of observation in UTC \\
\hline
\end{tabular}

\subsection{NGS File}

The most commonly used file format for legacy mode i.e., $\mathrm{S}$ and $\mathrm{X}$ band observation is NGS card [32]. It is mainly composed of an ASCII file with different cards based on the session and 
observation. Each file contains observables from a single band which means in case of dual band observation, two separate NGS card files are generated. The main objective of observing with dual frequency band is to remove propagation delay caused by the ionosphere. Short baseline measurements are never affected by this effect as both the telescopes are observing in the same part of the sky. Moreover, most of the scans in S-band are affected by RFI [33] mainly due to overlapping with Wi-Fi and telecommunication signal. This causes a hindrance in terms of assessing the data quality of the affected channels as they have to be flagged in the correlation process and are not contributing to further analysis. Hence, for short baseline measurements group delays only from X-band are used in the analysis process. The format of an NGS file [34] is described in Table 7.

Table 7. NGS card file format.

\begin{tabular}{cc}
\hline Card Name & Description \\
\hline Header card & General information about the session \\
Site card & Participating station information \\
Radio source position & Observed source position \\
Auxiliary parameters & Ref. frequency, group delay ambiguity spacing, delay type and rate information \\
Data cards & Scan-wise information based on vex \\
\hline
\end{tabular}

\section{Method}

\subsection{Schedule Preparation}

The provided dataset was scheduled using VIE_SCHED module of VieVS 3.0. All the input files are similar to sked. List of input files with a brief explanation of the content is provided in Table 8. There are mainly two modes of scheduling i.e., automatic and manual. Automatic scheduling mode is used for scheduling most of the geodetic sessions. The short baseline observations were also scheduled using automatic mode. In this approach, the software automatically selects the sources depending on the parameters defined by the scheduler. On the other hand, in manual mode, the scheduler selects the source and participating baseline manually for each scan. This is very useful for performing pointing and calibration tests by selecting an appropriate source based on the requirement. The output is stored in ASCII format with the skd extension containing an observation plan. Along with the schedule file, VLBI experiment (VEX) file is also created for correlating the observed dataset. During the schedule preparation, the scheduler can also enable the schedule analyser menu. This tool helps in giving a review of the session as shown in Figure 7. The last column of Table 8 is indicating the type of catalogue. Most of the files are directly copied from the GSFC server and read by the software. For some special purposes, there are some local files as an indicator, e.g., param.txt is a file for the scheduler to define the scheduling parameters, snrmin.txt is created to add minimum SNR for particular telescopes etc.

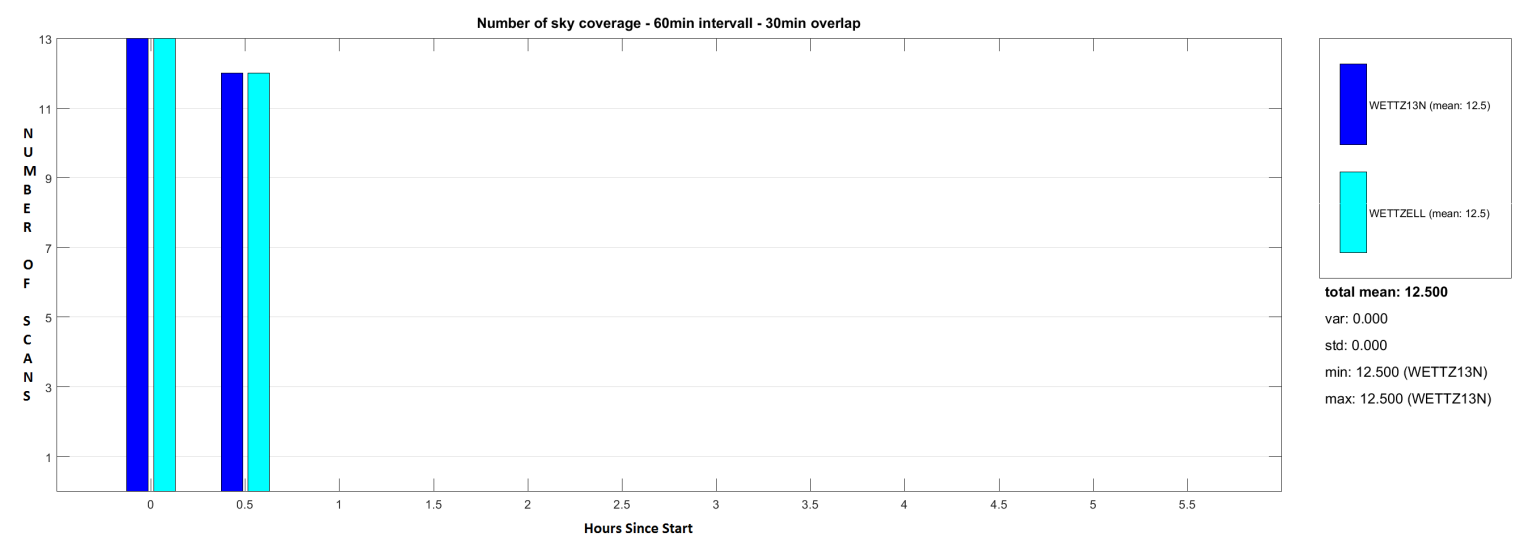

Figure 7. Sky-coverage histogram plot vs observation time for all the participating telescopes. 
Table 8. List of input files to the scheduling module.

\begin{tabular}{ccc}
\hline File Name & Description & Type \\
\hline Source.cat & Source location catalogue & GSFC \\
Flux.cat & Source flux catalogue & GSFC \\
Antenna.cat & Site catalogue & GSFC \\
Position.cat & Station location catalogue & GSFC \\
Equip.cat & Hardware description catalogue & GSFC \\
Mask.cat & Elevation mask catalogue & GSFC \\
Modes.cat & Measurement mode catalogue & GSFC \\
Freq.cat & Frequency setup & GSFC \\
rx.cat & Receiving unit & GSFC \\
loif.cat & Local oscillator frequency & GSFC \\
rec.cat & Data recording setup & GSFC \\
hdpos.cat & Head position setup & GSFC \\
tracks.cat & Recording tape setup & GSFC \\
param.txt & Scheduling parameter definition file & Local \\
down.txt & Define availability of station & Local \\
snrmin.txt & Minimum signal to noise ratio & Local \\
Psource.txt & Particular source file mainly for astronomy & local \\
\hline
\end{tabular}

\subsection{The Observation}

The short baseline measurements are observed between 08:00 in the morning and 17:00 local time in the evening. The main reason is to avoid coincidence with the global session which in most of the cases start after 18:00 UT. These observations are also beneficial for scientific community as with the new VLBI2010 concept, having more than one telescope at a cite is foreseen as the future of Very long baseline observation. These local sessions are a very good starting point in terms of understanding the VLBI system from scheduling to geodetic parameter estimation stage.

Before the real observation takes place, the scheduler has to submit a request in a coordination tool. In terms of planning, there is a possibility that depending on the chosen baseline, the tool automatically provides a list of available days with duration (Figure 8). The available time slots are denoted by ' $\mathrm{F}^{\prime}$ whereas ' $x$ ' is an indicator of unavailability of the selected telescope. As a result, it is very convenient to add a new session and the requested session with the session notes describing the session is published on the designated website with the current status. After the session is requested, it is accepted or rejected depending on the operator's observation plan. The schedule files are generated and copied to the local FTP server for all the accepted session.

\subsection{Correlation}

The post-observation step is to send the raw data are to the correlation facility with the station $\log$. In case of short baseline observation between RTW and WN, raw data is transferred to the high speed data recorder and streamer (flexbuff) with total storage space of $100 \mathrm{~TB}$. It is connected to the computing cluster on which DiFX and HOPS package is installed as shown in Figure 9.

The local correlator GOWL serves three main purposes:

1. It is primarily designed for relative positioning of the three Wettzell radio telescopes, i.e., to derive the local ties between the three telescopes from VLBI raw data in addition to the conventional terrestrial surveys carried out.

2. The local correlator closes the gap between the observation work and geodetic analysis. The closure of this missing piece in the measurement/analysis chain is important to provide timely quality feedback to the VLBI engineers regarding the status of their telescopes.

3. Finally, GOWL serves as a critical backup infrastructure. This means that it is not foreseen to carry out routine operational work, which resides with the Bonn Correlator operated at the MPIfR, but is available in case of dedicated and special needs. 


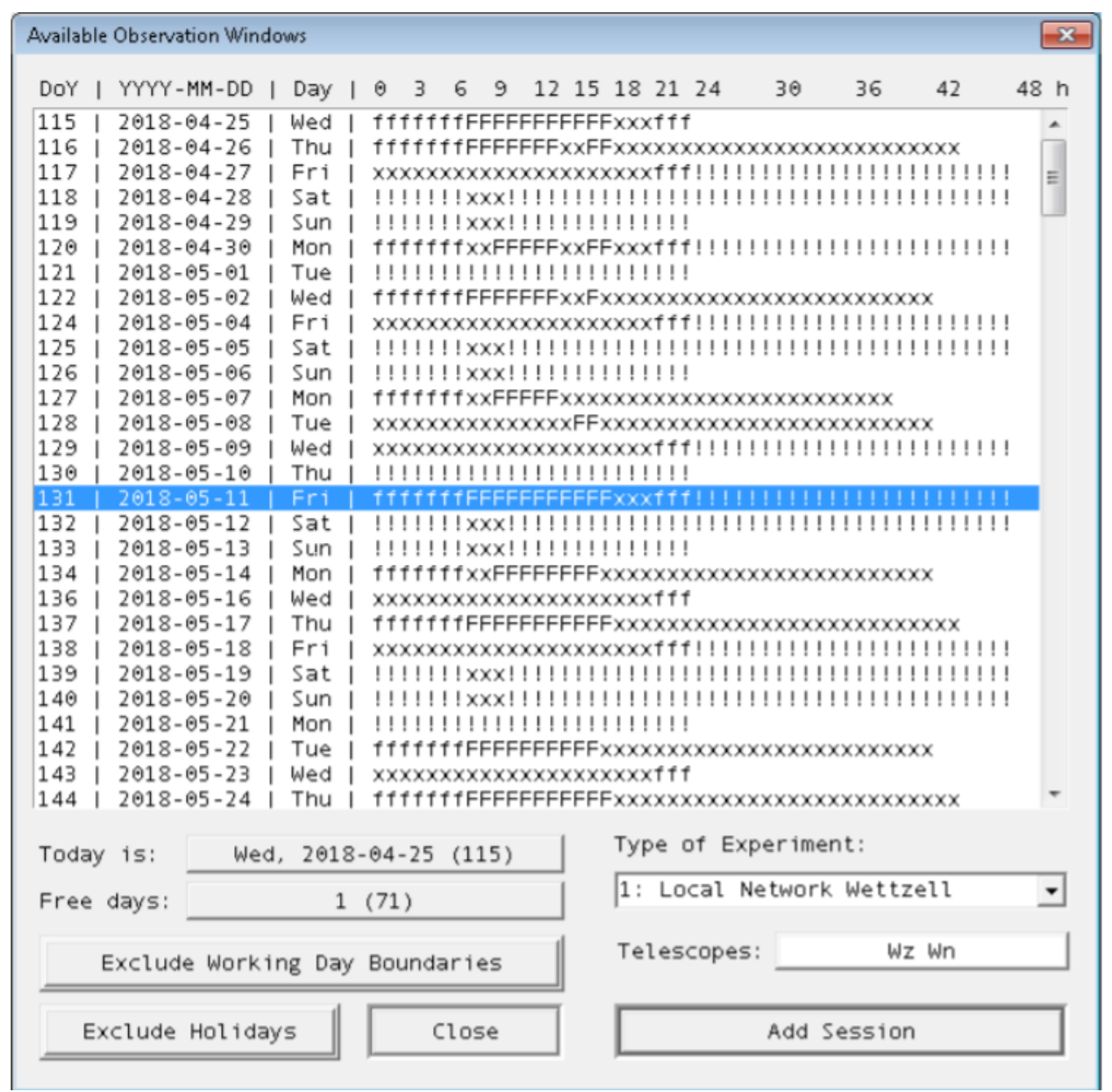

Figure 8. Screen shot of the coordination tool with the available time slots for Wn-Wz baseline.
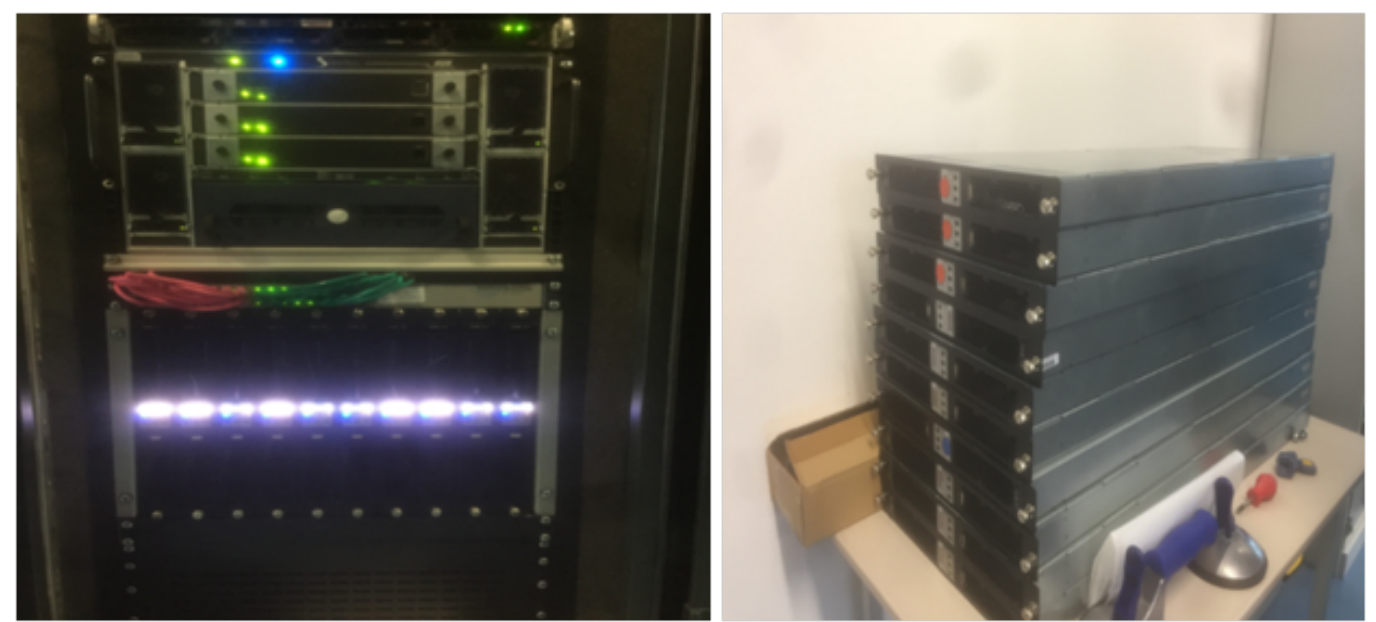

Figure 9. Image of the computing cluster installed at the TWIN operations room with the spare nodes.

\subsection{Postprocessing and Analysis}

The correlated (SWIN) dataset is processed in the fringe fitting software (HOPS). Fringe fitting accounts for insufficiencies in the correlator model and integrates the interferometer signal. Another advantage is the possibility to generate fringe plots using fourfit program for quality control. Fourfit is a part of HOPS package developed at Haystack Observatory, USA. Fringe plots are generated and read by the correlation analyst for detecting problems at the VLBI site. 
Figure 10 can be used for explaining the fourfit plot where local baseline participated. It is produced from X-band observation with RHCP for the source $1128+385$. In the first row is the multi-band delay (blue) and rate plot (red). Multi-band delay is defined as the gradient of the phase of the visibility vs observed frequency band whereas multi-band delay rate is given by the rate of change of fringe from the centre of the delay trace due to errors in the models used for data correlation. Under the multi-band delay, there are two other plots. On the left is the amplitude of single band delay versus time and on the right is the power spectrum plot including both upper and lower bands. In the next row, the image of the amplitude and phase versus time for each BBC can be found. Pcal amplitude versus time is also shown for the participating baseline. General information about the scan is written on the right side. Apriori group delay, phase delay, group and phase delay residuals are also documented at the end. The HOPS output is read by the pre-processing software for including additional information e.g, resolving ambiguity, cable delay, and meteorological information. All this information is written in an ASCII file (NGS) for final processing. The group delay information only from X-band is written in the ASCII file. For geodetic parameter estimation, an in-house analysis software specially designed for short baseline purposes is used. Further information can be found in [35]. The estimated baseline components X, Y, Z, and 3-D distance are is plotted in Figures 11-14 respectively. The statistics can be found in Table 9 .

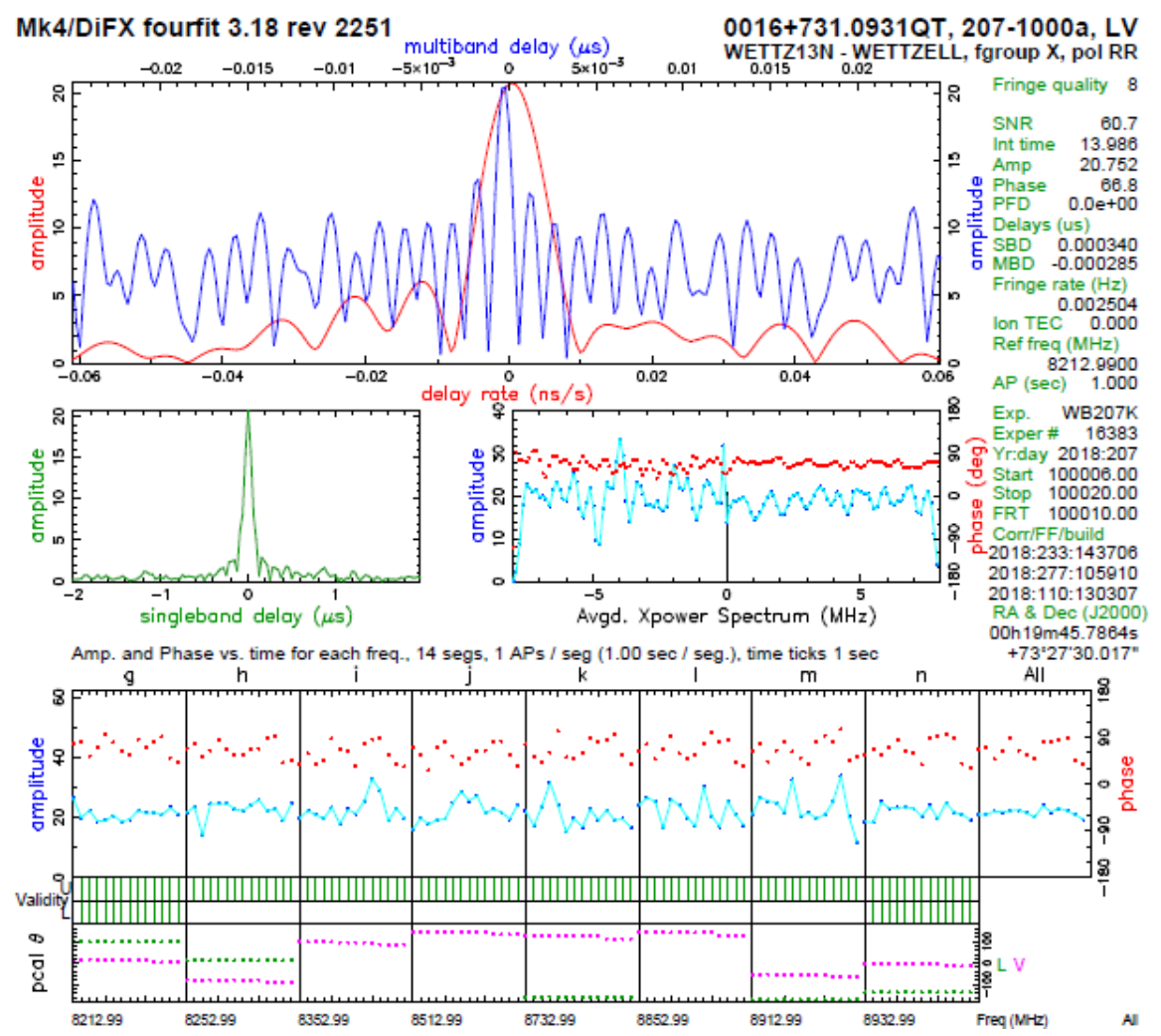

Figure 10. Example of a fringe plot from a local session.

Table 9. Analysis results from sessions observed in 2017.

\begin{tabular}{cccc}
\hline Baseline Component & Local Survey Measurement (m) & VLBI Analysis (m) & Offset (mm) \\
\hline X & -88.0363 & -88.0359 & 0.4 \\
Y & -38.7315 & -38.7313 & 0.2 \\
Z & 77.1628 & 77.1625 & 0.3 \\
3-D & 123.3070 & 123.3064 & 0.6 \\
\hline
\end{tabular}




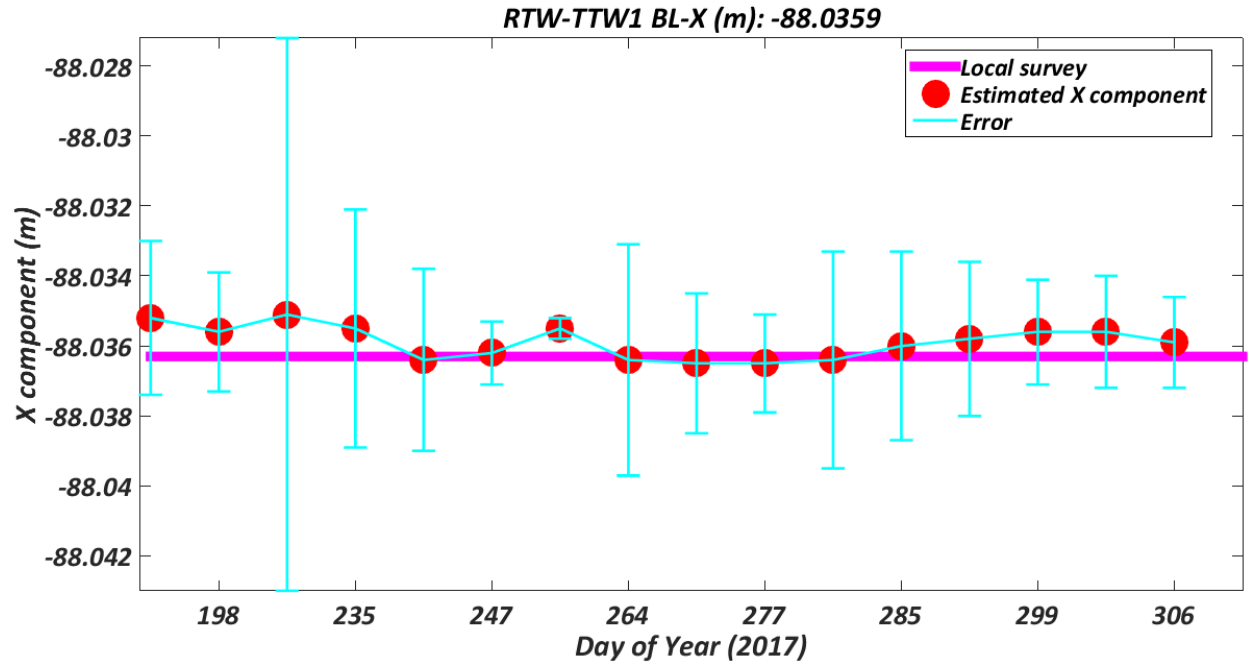

Figure 11. Estimated X-component from short baseline observations in 2017.

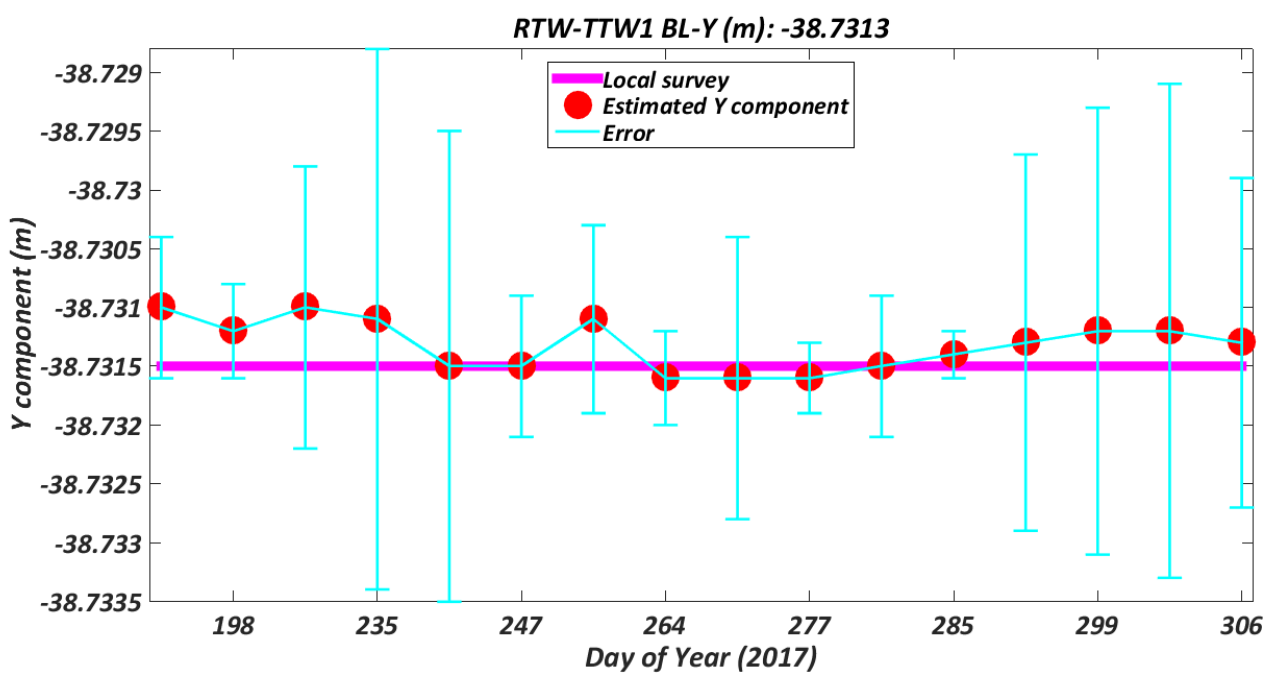

Figure 12. Estimated Y-component from short baseline observations in 2017.

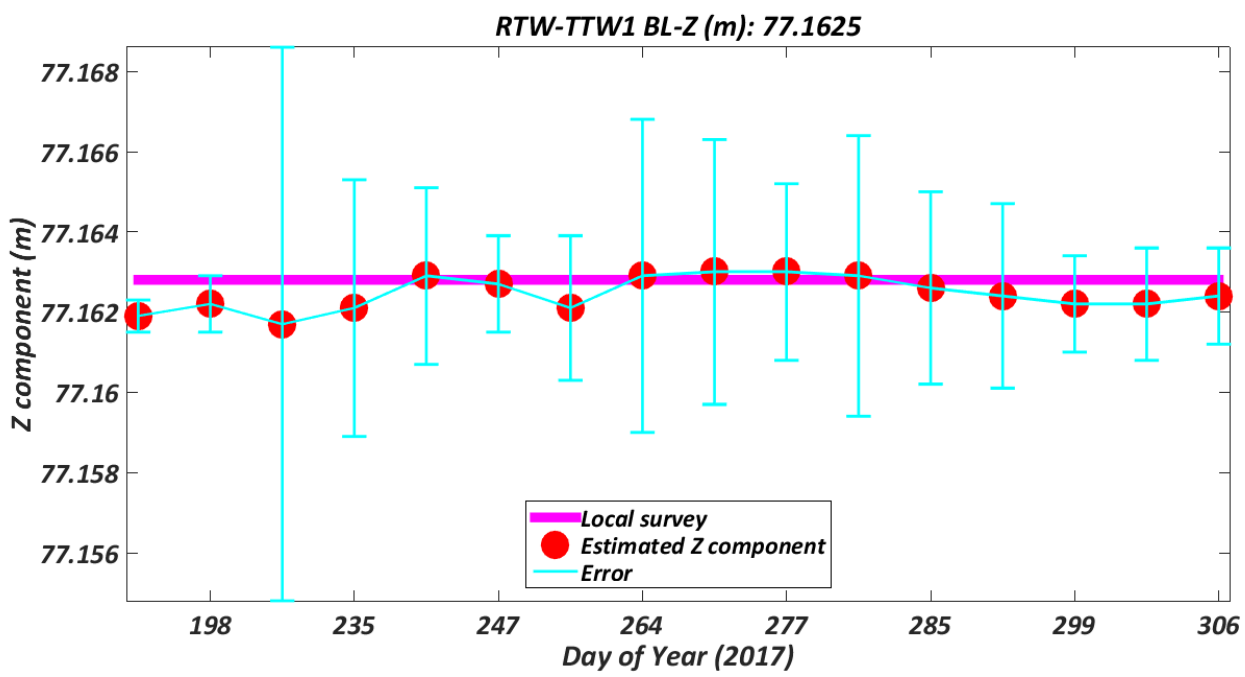

Figure 13. Estimated Z-component from short baseline observations in 2017. 


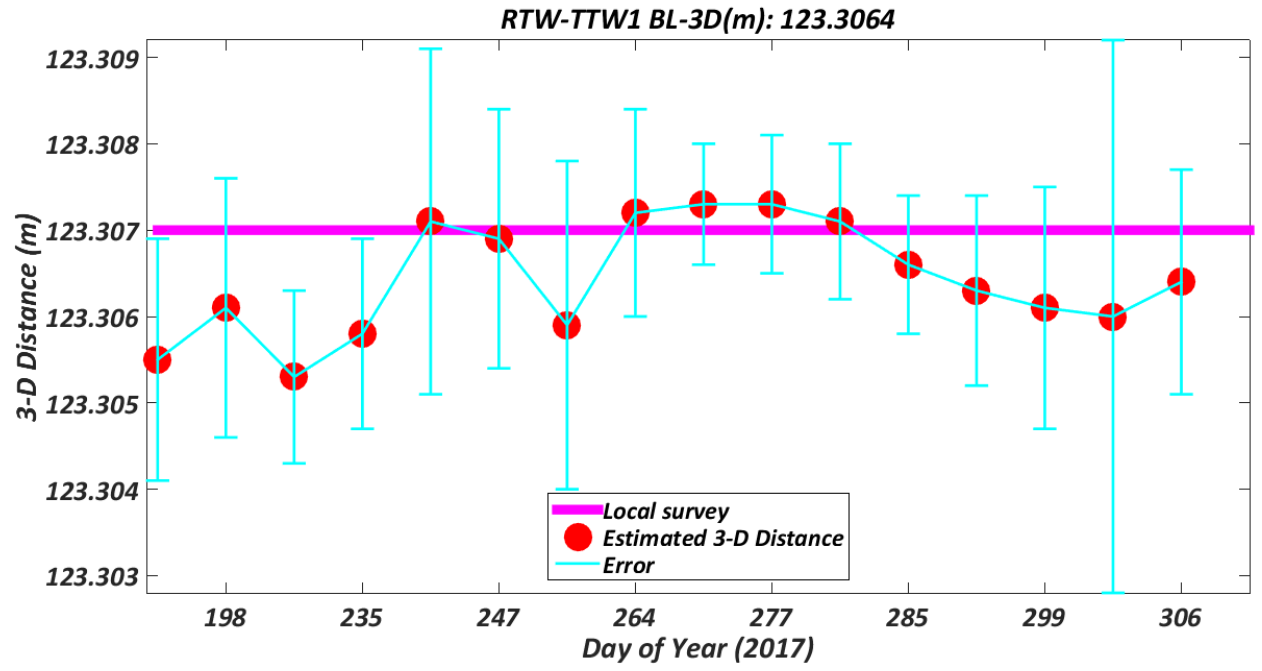

Figure 14. Estimated 3-D distance from short baseline observations in 2017.

\section{User Notes}

The published dataset is composed of 26 successfully analysed short baseline sessions. Each folder contains a number of files necessary for processing a geodetic session. The content of the provided dataset is given in Table 10 .

Table 10. List of files provided in the dataset.

\begin{tabular}{ccc}
\hline File/ Folder Name & Description & File Format \\
\hline sessionname.skd & Schedule file & ASCII \\
sessionnamesiteid.log & Station log & ASCII \\
sessionname.vex & VLBI experiment file & ASCII \\
alist.out & fringe fitting output & ASCII \\
vgosDB & VGOS database & ASCII and NetCDF \\
NGS File & group delay information & ASCII \\
\hline
\end{tabular}

A sessionname.skd is provided by the scheduler or P.I. to the operator for performing the observation, e.g., wb248i.skd where wb248i is the name of the schedule. During the observation, each station generates station log listed as sessionnamesiteid.log where siteid is added at the end of schedule name as an identifier, e.g., wb248iwn.log where wn is the site identifier. For the correlation process, the VLBI experiment file (sessionname.vex) is used. It is a single file with information about all the participating telescopes. Due to storage constrains the only alist.out is stored at the end of correlation and fringe fitting process where scan-wise details can be found. Inside session name folder there is another folder, e.g., 17SEP05X9 where vgosDB/netCDF output is stored with the wrapper and head files. The folder information can be used to process the VGOS database further using $v$ Solve. The vgosDB output provided in the dataset is version 1 . The final output from short baseline sessions is available in the NGS file. These files are generated after resolving ambiguity and adding meteorological data along with the group delay into the database for further processing using the in-house software.

Author Contributions: G.K., C.P. and W.S. performed the measurements, A.P. planned, scheduled, correlated and pre-processed the dataset, and T.S. supervised the dataset collection and analysed the pre-processed files (NGS card files) with the short baseline software (LEVIKA). A.P. wrote the manuscript with input from all co-authors.

Funding: This research received no external funding.

Acknowledgments: The research was conducted in the framework of LEVIKA (Local VLBI Correlation and Analysis) project funded by the Federal Agency for Cartography and Geodesy (BKG). The author would like to especially thank colleagues (Walter Alef and Helge Rottmann) from MPIfR for providing the hardware and necessary input for the installation of computing cluster and correlation software at GOW. We would 
also like to show our gratitude to the fellow employees (Laura La Porta and Simone Bernhart) from Reichert $\mathrm{GmbH}$ /Bundesamt für Kartographie und Geodäsie for sharing their pearls of wisdom with us during the course of this research. The author would like to gratefully acknowledge the software correlator (DiFX) used for correlating the dataset presented in this paper. The software correlator was developed at the Swinburne University of Technology as part of the Australian Major National Research Facilities Programme and operated under license. We thank the reviewers for their comments and suggestions.

Conflicts of Interest: The authors declare no conflict of interest.

\section{Abbreviations}

The following abbreviations are used in this manuscript:

VLBI Very Long Baseline Interferometry

IVS International VLBI Service for Geodesy and Astronomy

DiFX Distributed Fourier transform (F) and a cross-multiplication (X)

HOPS Haystack Observatory Postprocessing System

ASCII American Standard Code for Information Interchange

VieVS Vienna VLBI and Satellite Software

EOP Earth Orientation Parameter

NetCDF Network Common Data Form

GUI Graphical User interface

FS Field System

UPS Uninterruptible power supply

UT1 Universal Time

GPS Global Positioning System

vgosDB VLBI Global Observation System Data Base

VGOS VLBI Global Observation System

UTC Coordinated Universal Time

GSFC Goddard Space Flight Center

SNR Signal to Noise Ratio

FTP File Transfer Protocol

Gbps Gigabytes per second

RHCP Right Hand Circular Polarization

LEVIKA Local VLBI Korrelation and Analysis

MPIfR Max-Planck-Institute for Radio astronomy

DBBC Digital Base-Band Converter

LO Local Oscillator

BBC Base-Band Channel

IF Intermediate frequency

SEFD System Equivalent Flux Density

RFI Radio Frequency Interference

\section{References}

1. Maehler, S.; Schade, C.; Kluegel, T. Local ties at the Geodetic Observatory Wettzell. In Proceedings of the EGU General Assembly 2010, Vienna, Austria, 2-7 May 2010; Volume 12, p. 4815.

2. Neidhardt, A.; Kronschnabl, G.; Schatz, R. Geodetic Observatory Wettzell 20-m Radio Telescope and Twin Telescope. In International VLBI Service for Geodesy and Astrometry 2012 Annual Report; Behrend, D., Baver, K.D., Armstrong, K.L., Eds.; 2013; pp. 169-172. Available online: https://ivscc.gsfc.nasa.gov/ publications/ar2012/IVS-2012-Annual-Report.pdf (accessed on 27 November 2018).

3. International VLBI Service for Geodesy and Astrometry. Available online: https://ivscc.gsfc.nasa.gov /index. html (accessed on 27 November 2018).

4. Schüler, T.; Plötz, C.; Phogat, A. BKG CVC-The Central VLBI Observation Coordination Facility of the Federal Agency for Cartography and Geodesy at the Geodetic Observatory Wettzell. In Proceedings of the IVS General Meeting, Svalbard, Norway, 3-8 June 2018. 
5. Schüler, T.; Kronschnabl, G.; Plötz, C.; Neidhardt, A.; Bertarini, A.; Bernhart, S.; Porta, L.l.; Halsig, S.; Nothnagel, A. Initial results obtained with the first TWIN VLBI radio telescope at the Geodetic Observatory Wettzell. Sensors 2015, 15, 18767-18800. [CrossRef] [PubMed]

6. Kronschnabl, G.; Schüler, T.; Neidhardt, A.; Plötz, C.; Klügel, T.; Kodet, J. Status of the Wettzell Radio Telescope Triple and the O'Higgins VLBI System at Antarctica. In International VLBI Service for Geodesy and Astrometry 2014 General Meeting Proceedings: "VGOS: The New VLBI Network"; Behrend, D., Baver, K.D., Armstrong, K.L., Eds.; Science Press: Beijing, China, 2014; pp. 145-149. ISBN 978-7-03-042974-2.

7. Deller, A.T.; Tingay, S.; Bailes, M.; West, C. DiFX: A software correlator for very long baseline interferometry using multiprocessor computing environments. Publ. Astron. Soc. Pac. 2007, 119, 318-336. [CrossRef]

8. Deller, A.; Brisken, W.; Phillips, C.; Morgan, J.; Alef, W.; Cappallo, R.; Middelberg, E.; Romney, J.; Rottmann, H.; Tingay, S.; et al. DiFX-2: A more flexible, efficient, robust, and powerful software correlator. Publ. Astron. Soc. Pac. 2011, 123, 275-287. [CrossRef]

9. Rogers, A.E.; Cappallo, R.J.; Hinteregger, H.F.; Levine, J.I.; Nesman, E.F.; Webber, J.C.; Whitney, A.R.; Clark, T.A.; Ma, C.; Ryan, J.; et al. Very-long-baseline radio interferometry: The Mark III system for geodesy, astrometry, and aperture synthesis. Science 1983, 219, 51-54. [CrossRef] [PubMed]

10. Alef, W. Scheduling, Correlating, and Postprocessing of VLBI Observations. In Very Long Baseline Interferometry; Springer: Dordrecht, The Netherlands, 1989; pp. 97-139.

11. Gipson, J. An introduction to Sked. In Proceedings of the Sixth General Meeting of the International VLBI Service for Geodesy and Astrometry, Tasmania, Australia, 7-14 February 2010; pp. 77-84.

12. Gipson, J. Sked-VLBI Scheduling Software; NASA Goddard Space Flight Center: Greenbelt, MD, USA, 2012.

13. Sun, J.; Böhm, J.; Nilsson, T.; Krásná, H.; Böhm, S.; Schuh, H. New VLBI2010 scheduling strategies and implications on the terrestrial reference frames. J. Geod. 2014, 88, 449-461. [CrossRef] [PubMed]

14. Vandenberg, N. Drudg: Experiment Preparation Drudge Work; Space Geodesy Program, VLBI Software Manual; NASA/Goddard Space Flight Center: Greenbelt, MD, USA, 1997.

15. Himwich, E. Introduction to the field system for non-users. In International VLBI Service for Geodesy and Astrometry 2000 General Meeting Proceedings; National Aeronautics and Space Administration (NASA): Washington, DC, USA, 2000; pp. 86-90.

16. Bolotin, S.; Baver, K.; Gipson, J.; Gordon, D.; MacMillan, D. The VLBI Data Analysis Software nuSolve: Development Progress and Plans for the Future. In International VLBI Service for Geodesy and Astrometry 2014 General Meeting Proceedings: "VGOS: The New VLBI Network"; Behrend, D., Baver, K.D., Armstrong, K.L., Eds.; Science Press: Beijing, China, 2014; pp. 253-257. ISBN 978-7-03-042974-2.

17. Böckmann, S.; Artz, T.; Nothnagel, A.; Tesmer, V. International VLBI Service for Geodesy and Astrometry: Earth orientation parameter combination methodology and quality of the combined products. J. Geophys. Res. Solid Earth 2010, 115, B044404. [CrossRef]

18. Altamimi, Z.; Collilieux, X.; Métivier, L. ITRF2008: An improved solution of the international terrestrial reference frame. J. Geod. 2011, 85, 457-473. [CrossRef]

19. Böhm, J.; Böhm, S.; Boisits, J.; Girdiuk, A.; Gruber, J.; Hellerschmied, A.; Krásná, H.; Landskron, D.; Madzak, M.; Mayer, D.; et al. Vienna VLBI and satellite software (VieVS) for geodesy and astrometry. Public. Astron. Soc. Pac. 2018, 130, 044503. [CrossRef]

20. Vandenberg, N. Standard Schedule File Format, VLBI Software Documentation, Scheduling Program, Program Reference Manual, NASA/Goddard Flight Center, Space Geodesy Program. 1997. Available online: ftp:/ /ftp.ga.gov.au/geodesy-outgoing/vlbi/dele405/Jupiter/2011/SKED.pdf (accessed on 3 December 2018).

21. VEX File Definition/Example. Available online: https://vlbi.org/vex/docs/vex (accessed on 3 August 2018).

22. Field System (FS) Documents. Available online: https://vlbi.gsfc.nasa.gov/fsdoc/ (accessed on 1 December 2018).

23. Ambrosini, R.; Clark, T.; Corey, B.; Himwich, E. IVS Recommended Maser Timing Practices. 2014. Available online: https:/ /ivscc.gsfc.nasa.gov/publications/memos/ivs-2014-001v01.pdf (accessed on 27 November 2018).

24. Petrov, L. Instrumental errors of geodetic VLBI. In International VLBI Service for Geodesy and Astrometry 2000 General Meeting Proceedings; National Aeronautics and Space Administration (NASA): Washington, DC, USA, 2000; pp. 230-235.

25. Treuhaft, R.; Lanyi, G. The effect of the dynamic wet troposphere on radio interferometric measurements. Radio Sci. 1987, 22, 251-265. [CrossRef] 
26. Krügel, M.; Thaller, D.; Tesmer, V.; Rothacher, M.; Angermann, D.; Schmid, R. Tropospheric parameters: Combination studies based on homogeneous VLBI and GPS data. J. Geod. 2007, 81, 515-527. [CrossRef]

27. Haystack Observatory Postprocessing System. Available online: https://www.haystack.mit.edu/tech/vlbi/ hops.html (accessed on 31 July 2018).

28. Petrachenko, W.; Behrend, D.; Hase, H.; Ma, C.; Niell, A.; Schuh, H.; Whitney, A. The VLBI2010 global observing system (VGOS). In Proceedings of the EGU General Assembly EGU General Assembly 2013, Vienna, Austria, 7-12 April 2013.

29. Petrachenko, B.; Behrend, D.; Gipson, J.; Hase, H.; Ma, C.; MacMillan, D.; Niell, A.; Nothnagel, A.; Zhang, X. VGOS Observing Plan. In Proceedings of the 8th IVS General Meeting, VGOS: "The New VLBI Network"; Science Press: Beijing, China, 2014; pp. 16-19.

30. Gipson, J.; Böhm, J.; Bolotin, S.; Gordon, D.; Hobiger, T.; Jacobs, C.; Kurdobov, S.; Nothnagel, A.; Sovers, O.; Titov, O.; et al. Final Report of IVS Working Group 4 (WG4) on Data Structures. In International VLBI Service for Geodesy and Astrometry 2013 Annual Report; NASA/TP-2014-217522; NASA GSFC: Greenbelt, MD, USA, 2014; pp. 11-25.

31. Bolotin, S.; Baver, K.; Gipson, J.; Gordon, D.; MacMillan, D. Implementation of the vgosDb Format at the GSFC VLBI Analysis Center. In Proceedings of the 23rd European VLBI Group for Geodesy and Astrometry Working Meeting, Göteborg, Sweden, 14-19 May 2017; pp. 235-237.

32. Thorandt, V.; Wojdziak, R. BKG Data Center; NASA Goddard Space Flight Center: Greenbelt, MD, USA, 2008; p. 161.

33. Abidin, Z.Z.; Ibrahim, Z.A.; Rosli, Z.; Malim, S.F.F.; Anim, N.M.; Noorazlan, N. An RFI investigation for setting up a VLBI station below $2.8 \mathrm{GHz}$ in Malaysia. New Astron. 2012, 17, 61-64. [CrossRef]

34. NGS Card File Format. Available online: https://vlbi.gsfc.nasa.gov/global/ngs-doc.html (accessed on 25 October 2018).

35. Schüler, T.; Plötz, C.; Phogat, A. LEVIKA SBA-Wettzell Radio-Telescope Positioning with a Tailor-Made Analysis Software. In Proceesdings of the IVS General Meeting, Svalbard/Norwegen, Norway, 3-8 June 2018.

(C) 2018 by the authors. Licensee MDPI, Basel, Switzerland. This article is an open access article distributed under the terms and conditions of the Creative Commons Attribution (CC BY) license (http:/ / creativecommons.org/licenses/by/4.0/). 